\title{
Advantages of the avian model for human ovarian cancer (Review)
}

\author{
ANA DE MELO BERNARDO ${ }^{1}$, SÓLVEIG THORSTEINSDÓTTIR ${ }^{2}$ and CHRISTINE L. MUMMERY ${ }^{1}$ \\ ${ }^{1}$ Department of Anatomy and Embryology, Leiden University Medical Center, 2333 ZC Leiden, The Netherlands; \\ ${ }^{2}$ Centre for Ecology, Evolution and Environmental Change, Department of Animal Biology, \\ Faculty of Sciences, University of Lisbon, 1749-016 Lisbon, Portugal
}

Received March 2, 2015; Accepted May 15, 2015

DOI: $10.3892 / \operatorname{mco} .2015 .619$

\begin{abstract}
Ovarian cancer (OC) is the most lethal gynecological cancer. Early detection of OC is crucial for providing efficient treatment, whereas high mortality rates correlate with late detection of $\mathrm{OC}$, when the tumor has already metastasized to other organs. The most prevalent type of OC is epithelial OC (EOC). Models that have been used to study EOC include the fruit fly, mouse and laying hen, in addition to human EOC cells in 3D culture in vitro. These models have helped in the elucidation of the genetic component of this disease and the development of drug therapies. However, the histological origin of EOC and early markers of the disease remain largely unknown. In this study, we aimed to review the relative value of each of the different models in EOC and their contributions to understanding this disease. It was concluded that the spontaneous occurrence of EOC in the adult hen, the prolific ovulation, the similarity of metastatic progression with that in humans and the advantages of using the chicken embryo for modelling the development of the reproductive system, renders the hen particularly suitable for studying the early development of EOC. Further investigation of this avian model may contribute to a better understanding of EOC, improve clinical insight and ultimately contribute to decreasing its mortality rates among humans.
\end{abstract}

\section{Contents}

1. Introduction

2. Pathology and origin of EOC in humans

3. Current EOC treatments

4. Animal models in EOC research

Correspondence to: Professor Christine L. Mummery, Department of Anatomy and Embryology, Leiden University Medical Center, Postal zone S-1-P, P.O. Box 9600, Building 2, Einthovenweg 20, 2333 ZC Leiden, The Netherlands

E-mail: c.1.mummery@lumc.nl

Key words: avian, ovarian cancer, ovary, Fallopian tube, cancer modelling
5. The domestic hen: A unique model to study EOC

6. Genetic tools: Boosting avian models in EOC

7. The avian model as a key player in EOC research

\section{Introduction}

Ovarian cancer (OC) is the fifth most common cause of cancer-related mortality in women. A high incidence of OC correlates geographically with more economically developed countries $(1,2)$. The current treatments for OC are cytoreductive surgery and platinum/paclitaxel ( Taxol ${ }^{\circledR}$ )-based chemotherapy (3). These therapies are efficient in the treatment of $90 \%$ of patients diagnosed with OC, but only when the disease is detected at an early stage (4). In addition, the treatments lack specificity, further contributing to the high mortality rates of OC $(3,5,6)$. The absence of an anatomical barrier around the ovary facilitates rapid spreading of metastases in the peritoneal cavity and late diagnosis is attributed to the minimal manifestations of early $\operatorname{EOC~}(4,7)$. As a consequence, the majority of OC cases are detected only when the cancer has already metastasized to other anatomical structures (8).

Epithelial OC (EOC) is the most common type of OC, constituting $90 \%$ of diagnosed OC cases (9). One of the greatest challenges in EOC research is to understand its cellular and molecular origin(s) (10). Different in vivo and in vitro systems have been used to model EOC. Drosophila melanogaster (D. melanogaster) and Mus musculus (M. musculus) EOC models have been helpful in elucidating the biological characteristics of EOC, such as the molecular basis of its metastatic mechanisms, which include alterations in cell adhesion or migration, or expression of genes involved in EOC development (11,12). Unlike humans, however, neither flies nor mice spontaneously develop EOC; therefore, the translation of outcomes to humans is limited. By contrast, in vitro EOC models using human cells are a promising approach to testing anticancer drugs, although the absence of the tumor cell microenvironment is associated with certain limitations (13). Gallus gallus domesticus, the domestic hen, is a model which appears to address some of these shortcomings and, with the recent advances in laboratory tools for chicken research, it is becoming a tractable system for the study of EOC (14). The hen is the only animal model that, like humans, develops the disease spontaneously and exhibits similar pathology and 
disease progression; this appears to be associated with prolific ovulation and ageing (14).

The aim of the present review was: i) To provide an overview of the current approaches and challenges in OC research, with a focus on EOC; ii) to provide a comparative analysis of the advantages and disadvantages of the different models used in EOC research; and iii) to investigate Gallus gallus domesticus as a model to answer fundamental questions regarding the origin of EOC that remain unanswered and to advance modalities for treatment and early diagnosis that may ultimately contribute to decreasing the mortality rate of OC.

\section{Pathology and origin of EOC in humans}

Pathogenesis. More than 30 types of OC have been described, which are all derived from only three major progenitor cell types, namely stromal cells, germ cells and surface epithelial cells (Fig. 1A). Stromal-cell OC (SCOC) results from the transformation of stromal cells present in the ovary and has a very low prevalence among OCs (7\%); germ-cell OC (GCOC) results from germ cell abnormalities that arise during development and is the rarest histotypic origin of $\mathrm{OC}$, with a prevalence of only 3\%; EOC is by far the most prevalent OC histotype origin, with a prevalence of $90 \%$ (9). EOC results from the abnormal development of epithelial cells and its origin is discussed in detail below. The formation of malignant cysts from malignant epithelial cells is currently considered to herald the pathological development of EOC. Malignant epithelial ovarian cells in the cysts undergo epithelial-to-mesenchymal transition (EMT), becoming motile and capable of invading other tissues (15). The progress of the metastatic process depends on the ability of these cells to survive and attach to other structures $(8,16)$.

Causality. There is currently no consensus regarding the origin of EOC and it is considered to either derive from malignant alterations of the ovarian surface epithelium (17), or from the abnormal development of the Fallopian tube epithelium (18). The complexity of EOC appears to indicate that the ovarian surface epithelium as well as the Fallopian tube epithelium are involved in the development of this disease (10). The ovarian surface epithelium as the origin for EOC is the oldest hypothesis and has been associated with the high frequency of ovulation in women (19-21). During each ovulation, this epithelium is disrupted when the mature oocyte is expelled from the ovary and inflammatory processes are then required to repair it $(17,22)$. During the repair process, a proportion of the cells detach and develop abnormalities, due to the DNA damage in response to inflammatory molecules, resulting in EOC (23). A role for hormones in the damage of the ovarian surface epithelium has also been suggested $(24,25)$. The observation that women who use progestin-estrogen oral contraceptives have a 30-60\% lower probability of developing EOC, further strengthens the hypothesis that the ovarian surface epithelium is the origin of EOC (26). However, female mice, which ovulate approximately 4 times more than a woman during their lifespan, do not develop this disease (27). It is possible that structural differences in the ovarian surface epithelium between mice and humans (27) allow mice to develop a form of 'resistance' against EOC development, despite their significantly higher ovulation rates. Interestingly, it is estimated that the number of ovulations of a 2-year-old hen is similar to the number of ovulations of a woman at menopause (28). The fact that the hen is the only animal model that develops spontaneous EOC suggests similarities in the role of ovulation in the development of the disease between hens and humans. On the other hand, EOC was recently associated with abnormalities of the Fallopian tube epithelium. The Fallopian tube epithelium has been proposed as an origin of EOC, since several proteins normally expressed by the oviduct, such as paired box 8 (PAX8) and cancer antigen (CA)-125, have been found to be expressed in EOC biopsies $(10,29)$. Moreover, it has been suggested that a genetic predisposition in Fallopian tube epithelial cells gives rise to EOC; this includes mutations in DNA damage repair genes, such as BRCA1 and BRCA2 and cell cycle regulators, such as P53 (30). However, since the ovarian surface epithelium and the Fallopian tube epithelium are contiguous, have a common early embryonic origin and are both affected by ovulation, it is difficult to distinguish whether one or both tissues are the origin of EOC (10).

\section{Current EOC treatments}

There are four main factors that impede early detection of EOC. First, the location of the ovaries deep in the pelvic cavity makes it difficult to detect the initial development of EOC through pelvic probing and imaging. However, certain technological advances in this field, such as ultrasound and fluorodeoxyglucose-positron emission tomography/computed tomography, allow for better imaging and earlier detection $(6,31)$. Second, EOC was until recently considered to be an asymptomatic disease. Certain attempts have been made to establish a symptom index for OC; the physical symptoms may include gastrointestinal, genitourinary and gynecological complaints. These symptoms are, however, variable among patients, so this issue has not been resolved $(4,7,32)$. Third, there are currently no early tumor markers for EOC that allow early diagnosis, or population screening and later management of the disease, or monitoring of treatment effectiveness (33). Finally, the spread of malignant carcinogenic cells in the pelvic cavity is facilitated by the absence of a physical barrier around the ovaries. This promotes the spread of EOC along other organs, such as the contralateral ovary, the uterus and the peritoneum (8).

Once EOC is diagnosed, the primary treatment is surgical removal of the tumor. The surgery is normally followed by platinum and Taxol chemotherapy, which impairs cancer cell survival. Platinum-based treatments contain chemical compounds that promote DNA crosslinking, inhibiting DNA repair and synthesis (34), while Taxol promotes the assembly of microtubules in an irreversible manner, preventing cell division and promoting apoptosis of cancer cells (35). Regrettably, the chemotherapeutic agents used against OC are very similar to those used in the 1970s, when platinum-based therapies were first used in OC treatment (3). Alternatives to platinum/Taxol chemotherapy are currently under investigation (6). These include targeting tumor angiogenesis using inhibitors of proangiogenic proteins, such as vascular endothelial growth factor receptor (VEGFR), platelet-derived growth factor 
receptor and angiopoietins; or targeting key elements in cell growth, such as epidermal growth factor receptor (EGFR), which is overexpressed in EOC cells, using tyrosine kinase inhibitors and monoclonal antibodies against the extracellular domain of EGFR (36-39). The majority of these treatments are being developed in animal models but, unfortunately, often fail in clinical trials (33), highlighting the shortcomings of the animal models for human diseases (40). As a consequence, the $\mathrm{OC}$ post-diagnosis survival rates at 1,3 and 5 years have not changed significantly over the last 20 years (1). Accumulating knowledge on the origin of EOC is crucial to tackling this disease in its early stages, through identifying predictive EOC biomarkers for diagnosis and improvement of therapy (Fig. 1B). For this purpose, it is essential to establish a reliable experimental model capable of capturing all the characteristics of EOC pathology and origin.

\section{Animal models in EOC research}

D. melanogaster. The conserved mechanisms of molecular signalling pathways between fruit flies and humans, in combination with the ability to conduct large-scale genetic screens, makes $D$. melanogaster an excellent model for understanding the basic signalling mechanisms underlying the progression of EOC. Studies in D. melanogaster have helped identify tumor suppressor genes and oncogenes involved in OC development (16). Border cells present in the fly's ovaries have been used as a model to study EMT, which is part of the cancer metastatic process (41). These studies have identified polarity markers in the epithelium, such as E-cadherin and myosin IV, which play a role in the deregulation of proliferation and cell invasion, similar to what happens in human EOC (11). EGFR and VEGFR are key regulators of border cell invasiveness and have been studied in the fruit fly, since they are also involved in EOC (11). The role of the Hippo signalling pathway has also been investigated in the fruit fly as a model for EOC. Interestingly, by overexpressing the Yes-associated protein component of this pathway, which is also overexpressed in human EOC, it has been possible to induce EOC in flies, demonstrating its significance in EOC tumorigenesis and conservation of the process in humans (42). Studying Hippo signalling in fruit flies has revealed the role of this pathway in tissue growth regulation, through programming cell death and cell fate, in flies and humans (11). However, D. melanogaster remains a less than ideal clinical translational model, since it displays reduced metastatic potential and lacks the complexity of the human physiology and human immune system (41).

M. musculus. Mice are the most widely used animals for human disease modelling. In addition to a number of conserved molecular and physiological pathways, mice display a large repertoire of genetic and laboratory tools, still unsurpassed by other laboratory species (43). Mouse models in EOC have been extensively used to investigate disease progression in humans and to develop anti-OC drugs. Several mouse models of EOC with different characteristics have been developed. In this review, we aimed to focus on the comparison of advantages and disadvantages of three major groups of mouse models in $\mathrm{OC}$ research, namely xenograft, syngeneic and genetically engineered mice.
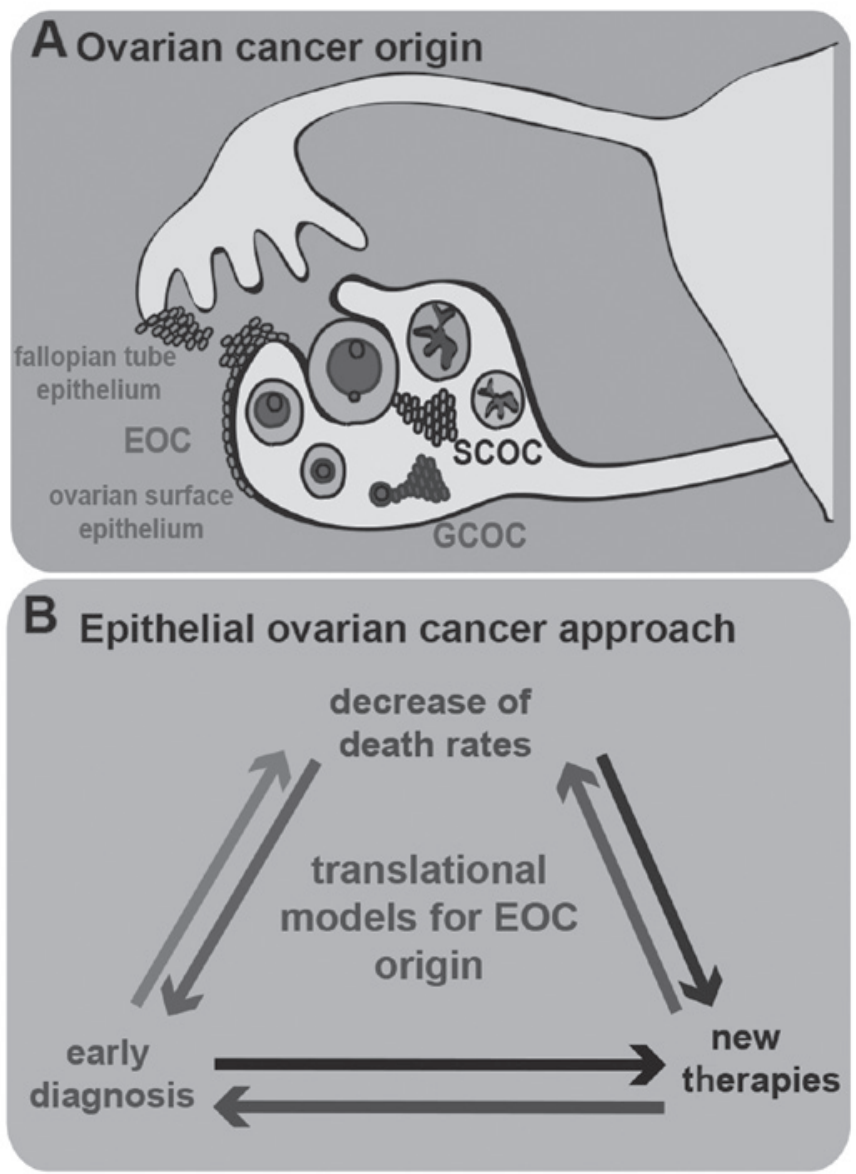

Figure 1. Ovarian cancer (OC) types and research approach in epithelial OC (EOC). (A) OC includes three major types: EOC, derived from the ovarian surface epithelium and/or the fallopian tube epithelium; germ-cell OC (GCOC), caused by abnormalities during germ cell development; and stromal-cell OC (SCOC), caused by abnormal development of stromal cells. (B) Establishing better translational models for the origin of EOC, the most prevalent type of OC, is fundamental to developing early diagnostic procedures and new therapies, thereby decreasing EOC death rates in humans.

Xenograft mouse models, in which human OC cells are introduced into host immunodeficient mice, enable the study of the early disease stages, as well as invasion and spreading of the cancer cells. These models have been used to evaluate therapeutic approaches, since they constitute a good representation of the disease and its heterogeneity $(44,45)$. The immune response, however, is completely absent in xenograft models, since the procedures are performed in immunodeficient mouse strains (43).

The development of syngeneic mouse models, in which the cancer cells are derived from the same mouse strain and are introduced into the immunocompetent host, overcome certain limitations of xenografts (46), although the EOC studied is mouse, rather than human. These models enable the study of immune response, tumor-secreting factors, epithelial-stromal interactions and tumor vascularization $(43,47)$.

Since the development of EOC in mice is never spontaneous and must always be induced (12), this is mostly achieved using genetically engineered mice (43). Mice have been engineered to overexpress genes associated with EOC in humans. These genes include P53, AKT, BRCA1 and BRCA2, 
which have been implicated in the progression and regression of this disease (48-51). However, the paucity of tissue-specific promoters for ovarian surface epithelium or Fallopian tube epithelium is a major limitation of this approach, since it is difficult to distinguish tissue-specific malignancy from the more general oncogenic properties of these genes (10). Nevertheless, engineered or transgenic mice have enabled the study of the effects of different mutations in EOC and the corresponding immune interactions $(12,43)$.

Taken together, these mouse models have overcome certain limitations of D. melanogaster in EOC research. However, they also present with their own biological limitations, which compromise their extrapolation to humans. For example, the heterogeneous origin of EOC requires its study in a heterogenetic background, which is not provided by inbred laboratory mouse strains. Moreover, EOC development in mice is not a spontaneous process, but rather induced as mentioned above, which, by definition, rules out the study of the origin and initial development of this disease, limiting the success of therapeutic response prediction in human patients. The development of new drugs using animal models requires a major investment from pharmaceutical companies, since only a limited number of these drugs continue to clinical trials. Failure to translate is a major obstacle towards finding cures for EOC (40).

In vitro models. In vitro systems, based particularly on human cell lines, are in principle an attractive alternative in terms of predictive power and also have the potential to be turned into high-throughput formats for therapeutic target identification. These in vitro systems may also capture patient genetic profiles, an important step in personalized medicine (13). This promise of bench-to-clinical translation has led to various attempts of developing reliable in vitro models of EOC. The current challenges are determining the best source of biomaterials and improving the culture conditions of EOC in order to mimic biological environments $(13,52)$. Unfortunately, cells derived from untreated tumors exhibit a tendency to develop drug resistance during primary culture using the presently available methods, limiting their value (53). Immortalized normal ovarian surface and/or Fallopian tube epithelia constitute promising alternatives, since they may be genetically modified and cultured for long periods, although they do not mimic the initial stages of the disease (53).

With regard to culture conditions, cell-spreading assays, where tumor cells spread on surfaces coated with extracellular matrix (ECM) proteins, have been used to study the migration of OC cells $(54,55)$. However, although these ECM proteins may also be present in the tumor, they do not mimic the tumor microenvironment in vivo. For this reason, 3D culture systems have been developed to provide a more appropriate microenvironment for EOC cells (56). 3D culture systems also allow other factors, such as oxygen tension, growth factor gradients and properties of the ECM, to be tightly controlled in order to test their effects on EOC development (57). However, despite the sophistication of these 3D systems, several widely used OC cell lines and immortalized ovarian surface or Fallopian tube epithelium lines have not been able to capture the biology of the tumor $(13,58)$. This issue has been associated with biomechanical and biophysical constraints and inappropriate ECM and, thus far, has not been resolved (59).
Several limitations, such as establishment of a proper ECM environment, absence of functional vasculature or cells that are able to mediate adaptive immune responses, remain to be overcome in order to construct truly representative EOC in vitro models (59). Improving in vitro models for EOC may be costly, due to the need for specialized materials and expertise, but is also dependent on a better understanding of the tumor microenvironment in vivo, which the $3 \mathrm{D}$ cultures attempt to mimic. This is presently considered to be 'a work in progress'.

\section{The domestic hen: A unique model to study EOC}

The female hen possesses a single functional ovary, which undergoes ovulation at a high rate during its lifespan (60). Despite anatomical differences, the laying hen is the only experimental model that develops spontaneous OC and, at the same time, offers the possibility of easy manipulation of external factors, such as nutrition or hormones and drug administration $(61,62)$. Moreover, the pathology and progression of the disease resembles that in humans in several respects $(63,64)$. Specific characteristics of the hen also overcome several limitations of the other models already discussed in the study of OC.

Incessant ovulation hypothesis. Fathalla (17) was the first to identify a possible association between the repeated involvement of ovarian surface epithelium in the process of ovulation and the frequency of the development of the common ovarian neoplasms from this epithelium. In his 'incessant ovulation hypothesis', Fathalla stresses the role of repeated repair of the ruptured ovarian surface epithelium in the induction of genetic aberrations in the tissue that culminate in the development of OC $(17,65)$. This hypothesis is in line with observations on the domestic hen, which ovulates daily, on average, for at least 2 years and exhibits an OC prevalence of 5-35\% among adult hens, depending on the genetic strain $(66,67)$. Moreover, the hypothesis relates EOC incidence in humans to the fact that modern women are generally exposed to a continuous ovulatory process from puberty to menopause. A continuous ovulatory process without fertilization results from the decreased pregnancy rates in modern society, also evidenced by the geographical co-localization of high OC incidence in more economically developed countries (17). There is strong evidence supporting an association between low prevalence of EOC and the use of oral contraceptives or/and pregnancy (65). While wild chickens may live for 20-30 years, the domestic hen has a relatively short lifespan and is subject to intense and concentrated egg production during the first 2 years of its life, which makes it an interesting model to study the role of ovulation in EOC. Indeed, Fathalla's theory laid the foundation for different studies regarding the role of ovulation in OC (17). The first study, using medroxyprogesterone, demonstrated decreased egg production and a $15 \%$ reduction in the incidence of EOC in 3-year-old birds (68). More recently, using progestin as contraceptive, a $90 \%$ decrease in OC incidence was achieved in treated hens compared with the controls (69). A short generation time, the possibility of controlling environmental factors and the availability of different genetic strains make the domestic hen a very useful model in chemoprevention experiments $(61,68,69)$. 
Biomolecular and metastatic traits. The similarities between the hen and humans with respect to EOC development are also observed in terms of pathology, with several similar histopathological subtypes identified in both species $(68,70)$. Moreover, the sequencing of the chicken genome 10 years ago enabled valuable molecular comparisons with human cases (71). Different biomarkers, such as CA-125, P53 and E-cadherin, were also expressed in EOC in both species (28,72-74).

With respect to the EOC origin, the same controversies apply to human and hens. In the hen, the expression of proteins that are specifically expressed in the oviduct during the later stages of the disease, such as ovoalbumin, ovostatin 2, PAX2 protein or EGFR1, indicate involvement of the oviduct in disease development $(10,72)$. This finding supports the involvement of the Fallopian tube epithelium in spontaneous EOC, as in humans. This trait makes the hen a particularly useful model to better understand the origin of the OC in humans, where the oviduct also appears to play a role $(63,67,75)$. Since, as mentioned earlier, female mice do not develop spontaneous EOC, the involvement of the Fallopian tube epithelium was only recently demonstrated: In a transgenic mouse model, in which SV40 large T-antigen was expressed under control of a mouse Müllerian-specific Ovgp-1 promoter, malignant progression of this epithelium was observed (76).

With respect to the pathology of EOC in the hen, this is a highly malignant cancer that metastasizes along the abdominal cavity, spreading to different organs within a short period of time (68). Histopathological evaluation of OC metastasis reveals similar characteristics between human and hen spontaneous adenocarcinomas of the reproductive tract. Interestingly, the metastatic process of EOC, in terms of the position and location of the ascites during the later stages of hen EOC, also resembles that in humans (18).

Despite significant evidence supporting the presence of similar molecular patterns in the origin and development of EOC, the lack of commercially available antibodies for immunohistochemistry and western blot analysis remains a major limitation in the use of hen models in EOC $(14,67)$. In order to increase the translational power of the laying hen as a model in OC research, it is crucial to develop further chicken laboratory tools in the fields of genomics, proteomics and metabolomics. These tools will likely be useful for the study of OC, as well as that of other pathologies (77).

Anatomy and heterogenetic background. Different EOC types display remarkable diversity at the cellular and molecular levels $(10,78)$. There is currently a scarcity of evidence regarding the role of specific genes in the development of EOC in humans, which appears to have heterogenic causes (78). The evidence indicating a heterogeneous background to EOC suggests that it is of paramount importance to establish an experimental model with a heterogenetic background to study this disease, rather than using inbred species (67). The domestic hen has been extensively bred for agricultural purposes, but its genome maintains the genetic diversity of the wild chicken (71). Studies regarding the role of ageing in the development of EOC in hens have demonstrated differences in EOC prevalence rates among different strains. Different strains appear to develop OC in parallel with ageing; however, the incidence rate of the disease differs among strains (66).
Development of the reproductive system. The fact that the hen develops in ovo, provides a significant advantage for in vivo manipulation and imaging of embryonic processes (79). The use of chicken embryos, which are amniotes, in cell interaction studies, cell fate tracing or mechanisms of embryonic patterning, has allowed investigation of several processes that have analogies in humans (79). The development of the urogenital system is a case in point, particularly with regard to understanding the signalling pathways underlying the development of the testes and ovaries (80). The development of the gonads in chickens displays one particularly striking characteristic: During gonadogenesis, the development of the gonads is asymmetric, resulting in two functional testes in males, but only one functional ovary on the left side in females (81). This asymmetric development of the chicken gonads affects gonadal morphology and the development of germ cells, as exemplified by the asymmetric expression of meiotic markers (unpublished data). In mammals, asymmetry between the two gonads is also established during development; this does not affect their functionality, as a pair of functional testes or ovaries form. However, this asymmetry becomes evident in the development of certain sexual differentiation disorders, such as hermaphroditism $(82,83)$. With respect to $\mathrm{OC}$, it is interesting to note that there appears to be a higher prevalence of GCOC in the right gonad compared to that in the left gonad. This asymmetric prevalence of GCOC suggests an association between this asymmetry and germ cell development (84). The chicken provides a model for asymmetric ovarian development, a mechanism that appears to play a role in germ cell development, which is affected in GCOC. Therefore, the higher prevalence of GCOC in the right ovary may be further elucidated by understanding the asymmetrical development of the gonads in the chicken. Regarding EOC, there is no evidence supporting a role for gonadal asymmetry in the prevalence of the disease in the right or left ovary (84); interestingly, however, paired-like homeodomain transcription factor 2 (PITX2), which is overexpressed in EOC, is also a key player in the asymmetric development of chicken female gonads $(85,86)$. The expression of PITX2 in the left gonad promotes proliferation of the left cortex, leading to the asymmetric development of the gonads $(85,87)$. Moreover, when induced in the left gonad, PITX2 promotes the formation of the right cortex (87). Interestingly, PITX2 plays an important role during development, but is normally silenced in the adult; its role in cancer was recently demonstrated in several tumor types, such as metastatic prostate cancer and breast cancer (88-91). The chicken embryo offers a unique experimental model to understand the role of PITX2 in gonadal development and the effects of the inhibition or overexpression of this transcription factor during development, which may provide insight into its role in the signalling pathways involved in the development of EOC.

\section{Genetic tools: Boosting avian models in EOC}

Since Aristotle, the first to study the avian model, the laying hen has been used extensively in experimental embryology, disease modelling and evolutionary studies (77). The hen has contributed to our understanding of numerous processes relevant to humans, including (abnormal) cardiac development 


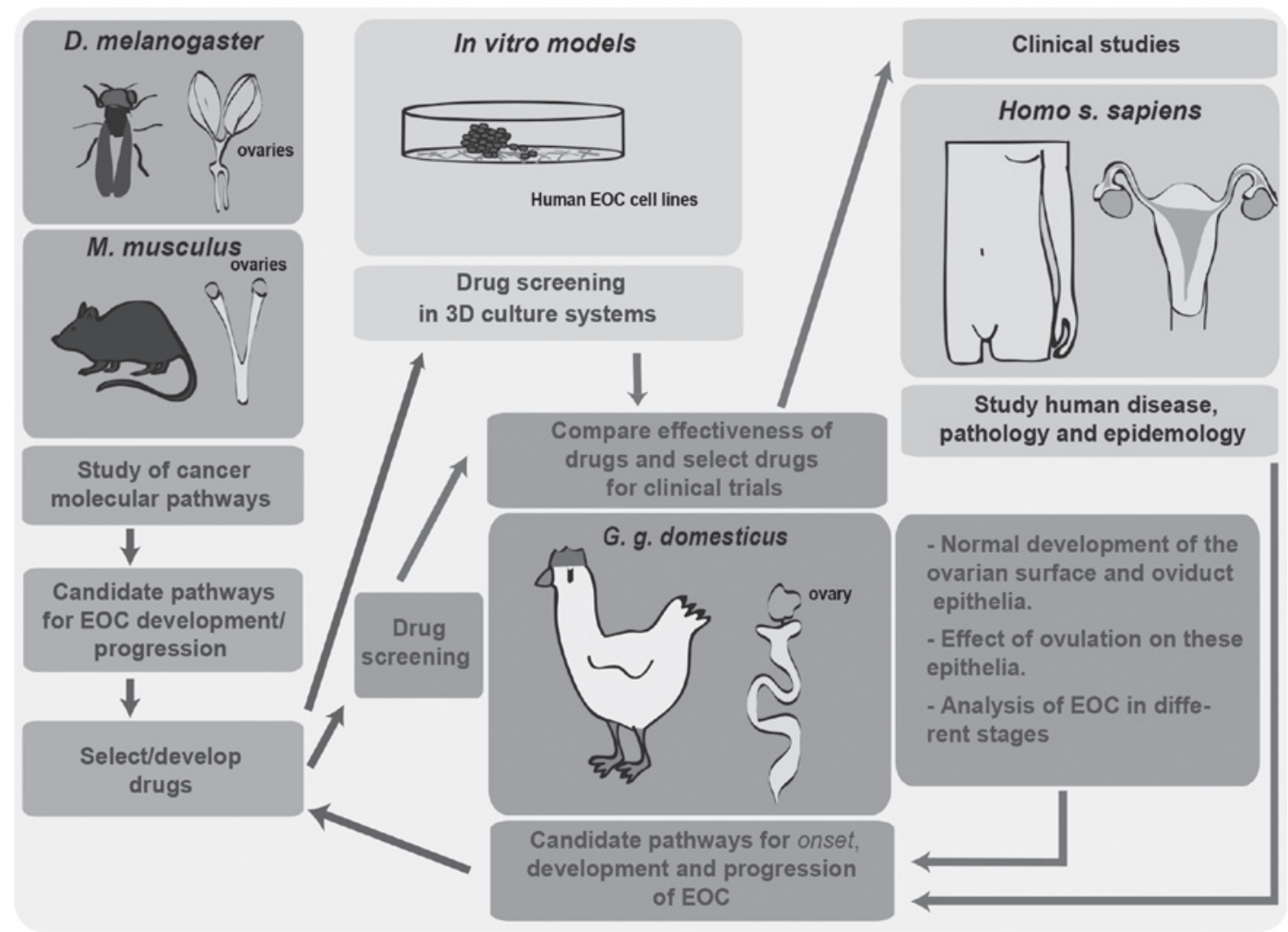

Figure 2. The role of the laying hen in an integrative epithelial ovarian cancer (EOC) research effort. Drosophila melanogaster (D. melanogaster) and Mus musculus (M. musculus) are well established models for the study of basic molecular mechanisms in cancer. Thus, they are excellent models to dissect out candidate pathways involved in EOC biology. However, D. melanogaster has limited clinical translation relevance, since it does not present the complexity of human physiology. M. musculus is physiologically very similar to humans, but does not provide a heterogenetic background and spontaneous development of EOC. 3D in vitro culture systems of human EOC cells may constitute an important model in drug screening, but they are not capable of reproducing the in vivo conditions. Gallus gallus domesticus is a promising model for EOC, not only by offering advantages compared with $M$. musculus in terms of in vivo drug validation, but is also a unique model for understanding the biological mechanisms underlying the onset, development and progression of EOC.

and somitogenesis, through which much of the skeletal musculature is formed (77). The differences between birds and humans, that may complicate EOC modelling, stem from the endocrine system and relate to the sexual hormone cycle $(14,69)$. However, the drawbacks of avian models in studying the origin of EOC are mostly associated with the lack of technology that provides appropriate laboratory tools (92). In contrast to flies and mice, there are few commercial sources of antibodies for immunohistochemistry, FACS or western blot analysis and transgenic approaches are only now becoming available in the chicken laboratories (92). Transgenesis in chicken is progressing slowly, despite the publication of the chicken genome sequence (71). Nevertheless, small interfering RNA and morpholino oligonucleotides have already been tested successfully in the avian model, allowing gain- and loss-of-function gene studies that are controlled in space and time (93). The development of isolation and culture methods of chicken embryonic stem cells has opened new doors in exploring chicken cell biology (94); however, as the available protocols are far from producing the first avian knockouts, it is currently necessary to rely on data from other models. New genetic tools, associated with its extensive history as an experimental model and low costs of acquisition and maintenance compared to other models, predict remarkable advances in the use of the hen for disease modelling $(77,92)$.
For EOC, long-term studies using the appropriate tools with regard to gene and protein expression will soon become more accessible. Together with the possibility of controlling gene expression and culturing chicken cells, these will allow researchers to investigate the spontaneous origin of EOC in a heterogenetic background and overcome certain of the limitations of other models.

\section{The avian model as a key player in EOC research}

Highlighting the advantages of the hen in studying the origin of EOC does not minimize the importance of improving other EOC models in parallel, but rather warrants the development of an integrative approach using different models, in vivo and in vitro, that may complement the discoveries made in the avian model (Fig. 2). Fruit fly and mouse models in EOC research will continue to unravel the basic mechanisms in EOC development and allow the development/selection of drugs that may be screened in 3D culture systems of human EOC cells. Subsequently, the hen offers the possibility of large-scale drug screenings in heterogeneous populations, enabling the comparison of drug efficiency in a robust model in order to better select drugs for clinical trials.

On the other hand, the laying domestic hen represents a unique system that mimics the disease in humans with regard 
to origin, development, metastatic processes and association with the ageing oviduct epithelium; in addition, the characterization of EOC in different progression stages in the adult hen may elucidate the mechanisms underlying the origin of EOC in humans. Therefore, the hen constitutes a fundamental model for the identification of candidate pathways associated with the onset, development and progression of EOC and the selection of drugs that target cancer pathways. Those drugs may be screened in other models, such as fruit fly, mouse and in vitro systems, but also in the hen itself. The complementary study of the different models may help us elucidate the pathology and epidemiology of this disease.

In conclusion, only an integrative research effort, where the avian model plays a crucial role, will enable the identification of new markers, thereby allowing the development of novel diagnostics and therapies for $\mathrm{OC}$, which remains the most common cause of gynecological cancer-related mortlaity in humans.

\section{Acknowledgements}

This study was supported by the Fundação para a Ciência e a Tecnologia SFRH/BD/94387/2013 to A.M.B.

\section{References}

1. Lowe KA, Chia VM, Taylor A, et al: An international assessment of ovarian cancer incidence and mortality. Gynecol Oncol 130: 107-114, 2013.

2. Siegel R, Ma JM, Zou ZH and Jemal A: Cancer Statistics, 2014 CA Cancer J Clin 64: 9-29, 2014.

3. Vaughan S, Coward JI, Bast RC, et al: Rethinking ovarian cancer: recommendations for improving outcomes. Nat Rev Cancer 11: 719-725, 2011

4. Bharwani N, Reznek RH and Rockall AG: Ovarian cancer management: the role of imaging and diagnostic challenges. Eur J Radiol 78: 41-51, 2011.

5. Luvero D, Milani A and Ledermann JA: Treatment options in recurrent ovarian cancer: latest evidence and clinical potential. Ther Adv Med Oncol 6: 229-239, 2014.

6. Menon U, Griffin M and Gentry-Maharaj A: Ovarian cancer screening - current status, future directions. Gynecol Oncol 132: 490-495, 2014.

7. Goff BA, Mandel LS, Drescher CW, et al: Development of an ovarian cancer symptom index: possibilities for earlier detection. Cancer 109: 221-227, 2007.

8. Bast RC, Hennessy B and Mills GB: The biology of ovarian cancer: new opportunities for translation. Nat Rev Cancer 9: 415-428, 2009

9. Romero I and Bast RC Jr: Minireview: human ovarian cancer: biology, current management, and paths to personalizing therapy. Endocrinology 153: 1593-1602, 2012.

10. King SM and Burdette JE: Evaluating the progenitor cells of ovarian cancer: analysis of current animal models. BMB Rep 44: 435-445, 2011

11. Rosales-Nieves AE and Gonzalez-Reyes A: Genetics and mechanisms of ovarian cancer: parallels between Drosophila and humans. Semin Cell Dev Biol 28: 104-109, 2014.

12. Fong MY and Kakar SS: Ovarian cancer mouse models: a summary of current models and their limitations. J Ovarian Res 2: 12, 2009.

13. Jacob F, Nixdorf S, Hacker NF and Heinzelmann-Schwarz VA: Reliable in vitro studies require appropriate ovarian cancer cell lines. J Ovarian Res 7: 60, 2014.

14. Johnson PA and Giles JR: The hen as a model of ovarian cancer. Nat Rev Cancer 13: 432-436, 2013.

15. Vergara D, Merlot B, Lucot JP, et al: Epithelial-mesenchymal transition in ovarian cancer. Cancer Lett 291: 59-66, 2010.

16. Naora $\mathrm{H}$ and Montell DJ: Ovarian cancer metastasis: integrating insights from disparate model organisms. Nat Rev Cancer 5 355-366, 2005.
17. Fathalla MF: Incessant ovulation - a factor in ovarian neoplasia? Lancet 2: 163, 1971.

18. Erickson BK, Conner MG and Landen CN Jr: The role of the Fallopian tube in the origin of ovarian cancer. Am J Obstet Gynecol 209: 409-414, 2013.

19. Fleming JS, Beaugie CR, Haviv I, Chenevix-Trench G and Tan OL: Incessant ovulation, inflammation and epithelial ovarian carcinogenesis: revisiting old hypotheses. Mol Cell Endocrinol 247: 4-21, 2006.

20. Murdoch WJ and McDonnel AC: Roles of the ovarian surface epithelium in ovulation and carcinogenesis. Reproduction 123: 743-750, 2002.

21. Murdoch WJ: Ovarian surface epithelium, ovulation and carcinogenesis. Biol Rev Camb Philos Soc 71: 529-543, 1996.

22. Auersperg N, Wong AS, Choi KC, Kang SK and Leung PC: Ovarian surface epithelium: biology, endocrinology, and pathology. Endocr Rev 22: 255-288, 2001.

23. Maccio A and Madeddu C: Inflammation and ovarian cancer. Cytokine 58: 133-147, 2012

24. Salehi F, Dunfield L, Phillips KP, Krewski D and Vanderhyden BC: Risk factors for ovarian cancer: an overview with emphasis on hormonal factors. J Toxicol Environ Health B Crit Rev 11: 301-321, 2008.

25. De Sousa Damião R, Fujiyama Oshima CT, Stávale JN and Gonçalves WJ: Analysis of the expression of estrogen receptor, progesterone receptor and chicken ovalbumin upstream promoter-transcription factor I in ovarian epithelial cancers and normal ovaries. Oncol Rep 18: 25-32, 2007.

26. Moorman PG, Havrilesky LJ, Gierisch JM, et al: Oral contraceptives and risk of ovarian cancer and breast cancer among high-risk women: a systematic review and meta-analysis. J Clin Oncol 31: 4188-4198, 2013.

27. Auersperg N: Specific keynote: experimental models of epithelial ovarian carcinogenesis. Gynecol Oncol 88: S47-51; discussion S52-55, 2003

28. Hakim AH, Turbov J and Rodriguez G: Ovarian adenocarcinomas in the laying hen and women share similar alterations in $\mathrm{p} 53$, ras, and HER-2/neu. Cancer Prev Res (Phila) 2: 114-121, 2009.

29. Kolwijck E, Span PN, Thomas CM, et al: Prognostic value of CA 125 in ovarian cyst fluid of patients with epithelial ovarian cancer. Oncol Rep 23: 579-584, 2010.

30. Walsh T, Casadei S, Lee MK, et al: Mutations in 12 genes for inherited ovarian, Fallopian tube, and peritoneal carcinoma identified by massively parallel sequencing. Proc Natl Acad Sci USA 108: 18032-18037, 2011.

31. Grant P, Sakellis C and Jacene HA: Gynecologic oncologic imaging with PET/CT. Semin Nucl Med 44: 461-478, 2014.

32. Chene G, Penault-Llorca F, Robin N, et al: Early detection of ovarian cancer: Tomorrow? A review. J Gynecol Obstet Biol Reprod (Paris) 42: 5-11, 2013 (In French).

33. Lokshin AE: The quest for ovarian cancer screening biomarkers: Are we on the right road? Int J Gynecol Cancer 22 (Suppl 1): S35-S40, 2012.

34. Muggia F: Platinum compounds 30 years after the introduction of cisplatin: implications for the treatment of ovarian cancer. Gynecol Oncol 112: 275-281, 2009.

35. Kumar S, Mahdi H, Bryant C, et al: Clinical trials and progress with paclitaxel in ovarian cancer. Int $J$ Womens Health 2: 411-427, 2010.

36. Huang RY, Chung VY and Thiery JP: Targeting pathways contributing to epithelial-mesenchymal transition (EMT) in epithelial ovarian cancer. Curr Drug Targets 13: 1649-1653, 2012.

37. Kalachand R, Hennessy BT and Markman M: Molecular targeted therapy in ovarian cancer: what is on the horizon? Drugs 71: 947-967, 2011.

38. Syrios J, Banerjee S and Kaye SB: Advanced epithelial ovarian cancer: from standard chemotherapy to promising molecular pathway targets - where are we now? Anticancer Res 34: 2069-2077, 2014.

39. Itamochi $\mathrm{H}$ and Kigawa J: Clinical trials and future potential of targeted therapy for ovarian cancer. Int J Clin Oncol 17: 430-440, 2012.

40. Caponigro G and Sellers WR: Advances in the preclinical testing of cancer therapeutic hypotheses. Nat Rev Drug Discov 10: 179-187, 2011.

41. Brumby AM and Richardson HE: Using Drosophila melanogaster to map human cancer pathways. Nat Rev Cancer 5: 626-639, 2005.

42. Hall CA, Wang R, Miao J, et al: Hippo pathway effector Yap is an ovarian cancer oncogene. Cancer Res 70: 8517-8525, 2010. 
43. House CD, Hernandez L and Annunziata CM: Recent technological advances in using mouse models to study ovarian cancer. Front Oncol 4: 26, 2014.

44. Shaw TJ, Senterman MK, Dawson K, Crane CA and Vanderhyden BC: Characterization of intraperitoneal, orthotopic, and metastatic xenograft models of human ovarian cancer. Mol Ther 10: 1032-1042, 2004.

45. Fu X and Hoffman RM: Human ovarian carcinoma metastatic models constructed in nude mice by orthotopic transplantation of histologically-intact patient specimens. Anticancer Res 13: 283-286, 1993.

46. Roby KF, Taylor CC, Sweetwood JP, et al: Development of a syngeneic mouse model for events related to ovarian cancer. Carcinogenesis 21: 585-591, 2000.

47. Urzua U, Roby KF, Gangi LM, et al: Transcriptomic analysis of an in vitro murine model of ovarian carcinoma: functional similarity to the human disease and identification of prospective tumoral markers and targets. J Cell Physiol 206: 594-602, 2006.

48. Orsulic S, Li Y, Soslow RA, et al: Induction of ovarian cancer by defined multiple genetic changes in a mouse model system. Cancer Cell 1: 53-62, 2002.

49. Xing D and Orsulic S: A mouse model for the molecular characterization of brcal-associated ovarian carcinoma. Cancer Res 66: 8949-8953, 2006.

50. Flesken-Nikitin A, Choi KC, Eng JP, Shmidt EN and Nikitin AY: Induction of carcinogenesis by concurrent inactivation of p53 and $\mathrm{Rb} 1$ in the mouse ovarian surface epithelium. Cancer Res 63: 3459-3463, 2003.

51. Wu R, Hendrix-Lucas N, Kuick R, et al: Mouse model of human ovarian endometrioid adenocarcinoma based on somatic defects in the Wnt/beta-catenin and PI3K/Pten signaling pathways. Cancer Cell 11: 321-333, 2007.

52. Fuller ES and Howell VM: Culture models to define key mediators of cancer matrix remodeling. Front Oncol 4: 57, 2014.

53. Gillet JP, Calcagno AM, Varma S, et al: Redefining the relevance of established cancer cell lines to the study of mechanisms of clinical anti-cancer drug resistance. Proc Natl Acad Sci USA 108: 18708-18713, 2011.

54. Yagi H, YotsumotoFand Miyamoto S: Heparin-binding epidermal growth factor-like growth factor promotes transcoelomic metastasis in ovarian cancer through epithelial-mesenchymal transition. Mol Cancer Ther 7: 3441-3451, 2008.

55. Sodek KL, Brown TJ and Ringuette MJ: Collagen I but not Matrigel matrices provide an MMP-dependent barrier to ovarian cancer cell penetration. BMC Cancer 8: 223, 2008.

56. Kenny HA, Krausz T, Yamada SD and Lengyel E: Use of a novel $3 \mathrm{D}$ culture model to elucidate the role of mesothelial cells, fibroblasts and extra-cellular matrices on adhesion and invasion of ovarian cancer cells to the omentum. Int J Cancer 121: 1463-1472, 2007.

57. Yamada KM and Cukierman E: Modeling tissue morphogenesis and cancer in 3D. Cell 130: 601-610, 2007.

58. Lee JM, Mhawech-Fauceglia P, Lee N, et al: A three-dimensional microenvironment alters protein expression and chemosensitivity of epithelial ovarian cancer cells in vitro. Lab Invest 93: 528-542, 2013.

59. White EA, Kenny HA and Lengyel E: Three-dimensional modeling of ovarian cancer. Adv Drug Deliv Rev 79-80: 184-192, 2014.

60. Pollock CG and Orosz SE: Avian reproductive anatomy, physiology and endocrinology. Vet Clin North Am Exot Anim Pract 5: 441-474, 2002.

61. Barnes MN, Berry WD, Straughn JM, et al: A pilot study of ovarian cancer chemoprevention using medroxyprogesterone acetate in an avian model of spontaneous ovarian carcinogenesis. Gynecol Oncol 87: 57-63, 2002.

62. Carver DK, Barnes HJ, Anderson KE, et al: Reduction of ovarian and oviductal cancers in calorie-restricted laying chickens. Cancer Prev Res (Phila) 4: 562-567, 2011.

63. Giles JR, Elkin RG, Trevino LS, et al: The restricted ovulator chicken: a unique animal model for investigating the etiology of ovarian cancer. Int J Gynecol Cancer 20: 738-744, 2010.

64. Seo HW, Rengaraj D, Choi JW, et al: The expression profile of apoptosis-related genes in the chicken as a human epithelial ovarian cancer model. Oncol Rep 25: 49-56, 2011.

65. Havrilesky LJ, Gierisch JM, Moorman PG, et al: Oral contraceptive use for the primary prevention of ovarian cancer. Evid Rep Technol Assess (Full Rep) 212: 1-514, 2013.

66. Johnson PA and Giles JR: Use of genetic strains of chickens in studies of ovarian cancer. Poult Sci 85: 246-250, 2006.
67. Hawkridge AM: The chicken model of spontaneous ovarian cancer. Proteomics Clin Appl 8: 689-699, 2014.

68. Fredrickson TN: Ovarian tumors of the hen. Environ Health Perspect 73: 35-51, 1987.

69. Trevino LS, Buckles EL and Johnson PA: Oral contraceptives decrease the prevalence of ovarian cancer in the hen. Cancer Prev Res (Phila) 5: 343-349, 2012.

70. Barua A, Bitterman P, Abramowicz JS, et al: Histopathology of ovarian tumors in laying hens: a preclinical model of human ovarian cancer. Int J Gynecol Cancer 19: 531-539, 2009.

71. International Chicken Genome Sequencing Consortium: Sequence and comparative analysis of the chicken genome provide unique perspectives on vertebrate evolution. Nature 432: 695-716, 2004.

72. Trevino LS, Giles JR, Wang W, Urick ME and Johnson PA: Gene expression profiling reveals differentially expressed genes in ovarian cancer of the hen: support for oviductal origin? Horm Cancer 1: 177-186, 2010.

73. Ansenberger K, Zhuge Y,Lagman JA, et al: E-cadherin expression in ovarian cancer in the laying hen, Gallus domesticus, compared to human ovarian cancer. Gynecol Oncol 113: 362-369, 2009.

74. Jackson E, Anderson K, Ashwell C, Petitte J and Mozdziak PE: CA 125 expression in spontaneous ovarian adenocarcinomas from laying hens. Gynecol Oncol 104: 192-198, 2007.

75. Lim CH, Lim W, Jeong W, et al: Avian WNT4 in the female reproductive tracts: potential role of oviduct development and ovarian carcinogenesis. PLoS One 8: e65935, 2013.

76. Kim J, Coffey DM, Creighton CJ, et al: High-grade serous ovarian cancer arises from Fallopian tube in a mouse model. Proc Natl Acad Sci USA 109: 3921-3926, 2012.

77. Kain KH, Miller JW, Jones-Paris CR, et al: The chick embryo as an expanding experimental model for cancer and cardiovascular research. Dev Dyn 243: 216-228, 2014.

78. Kurman RJ and Shih IeM: The origin and pathogenesis of epithelial ovarian cancer: a proposed unifying theory. Am J Surg Pathol 34: 433-443, 2010.

79. Davey MG and Tickle C: The chicken as a model for embryonic development. Cytogenet Genome Res 117: 231-239, 2007.

80. Ayers KL, Sinclair AH and Smith CA: The molecular genetics of ovarian differentiation in the avian model. Sex Dev 7: 80-94, 2013.

81. Smith CA and Sinclair AH: Sex determination: insights from the chicken. Bioessays 26: 120-132, 2004.

82. Mittwoch U: Phenotypic manifestations during the development of the dominant and default gonads in mammals and birds. J Exp Zool 281: 466-471, 1998.

83. Mittwoch U: Sex determination. EMBO Rep 14: 588-592, 2013.

84. Roychoudhuri R, Putcha V and Moller H: Cancer and laterality: a study of the five major paired organs (UK). Cancer Causes Control 17: 655-662, 2006.

85. Rodriguez-Leon J, Rodriguez Esteban C, Marti M, et al: PITX2 regulates gonad morphogenesis. Proc Natl Acad Sci USA 105: 11242-11247, 2008.

86. Fung FK, Chan DW, Liu VW, et al: Increased expression of PITX2 transcription factor contributes to ovarian cancer progression. PLoS One 7: e37076, 2012.

87. Guioli S and Lovell-Badge R: PITX 2 controls asymmetric gonadal development in both sexes of the chick and can rescue the degeneration of the right ovary. Development 134: 4199-4208, 2007.

88. Vela I, Morrissey C, Zhang X, et al: PITX2 and non-canonical Wnt pathway interaction in metastatic prostate cancer. Clin Exp Metastasis 31: 199-211, 2014.

89. Gobel G, Auer D, Gaugg I, et al: Prognostic significance of methylated RASSF1A and PITX2 genes in blood- and bone marrow plasma of breast cancer patients. Breast Cancer Res Treat 130: 109-117, 2011.

90. Wilting $\mathbf{J}$ and Hagedorn M: Left-right asymmetry in embryonic development and breast cancer: common molecular determinants? Curr Med Chem 18: 5519-5527, 2011.

91. Vandenberg LN and Levin M: A unified model for left-right asymmetry? Comparison and synthesis of molecular models of embryonic laterality. Dev Biol 379: 1-15, 2013.

92. Stern CD: The chick embryo - past, present and future as a model system in developmental biology. Mech Dev 121: 1011-1013, 2004.

93. Sauka-Spengler T and Barembaum M: Gain- and loss-of-function approaches in the chick embryo. Methods Cell Biol 87: 237-256, 2008.

94. Intarapat S and Stern CD: Chick stem cells: current progress and future prospects. Stem Cell Res 11: 1378-1392, 2013. 